\title{
miR-1299/NOTCH3/TUG1 feedback loop contributes to the malignant proliferation of ovarian cancer
}

\author{
YUQING PEI ${ }^{1}$, KEXIN LI $^{1}$, XIAOYING LOU ${ }^{1}$, YUE WU $^{1}$, XIN DONG $^{1}$, WENPENG WANG $^{2}$, \\ NING $\mathrm{LI}^{3}$, DONGHONG ZHANG ${ }^{4}$ and WEI CUI ${ }^{1}$ \\ ${ }^{1}$ State Key Laboratory of Molecular Oncology, Department of Clinical Laboratory, \\ National Cancer Center/National Clinical Research Center for Cancer/Cancer Hospital, \\ Chinese Academy of Medical Sciences and Peking Union Medical College, Beijing 100021; \\ ${ }^{2}$ Tianjin Medical University Cancer Institute and Hospital, National Clinical Research Center for Cancer, \\ Tianjin's Clinical Research Center for Cancer, Key Laboratory of Cancer Prevention and Therapy, Tianjin 300060; \\ ${ }^{3}$ Department of Gynecologic Oncology, National Cancer Center/National Clinical Research Center \\ for Cancer/Cancer Hospital, Chinese Academy of Medical Sciences and Peking Union Medical College, \\ Beijing 100021, P.R. China; ${ }^{4}$ Center for Molecular and Translational Medicine, Research Science Center, \\ Georgia State University, Atlanta, GA 30303, USA
}

Received November 21, 2019; Accepted April 29, 2020

DOI: $10.3892 /$ or.2020.7623

\begin{abstract}
Recent studies have revealed the oncogenic role of notch reporter 3 (NOTCH3) in ovarian cancer (OC). However, the possible regulators and mechanisms underlying notch receptor 3 (NOTCH3)-mediated behaviors in $\mathrm{OC}$ remain to be completely investigated. In the present study, we aimed to identify regulators of $\mathrm{NOTCH} 3$ and their interactions underlying the pathogenesis of OC. Bioinformatics analysis and luciferase reporter assay were used to identify potential regulatory miRNAs and lncRNAs of NOTCH3 in OC. Several in vivo and in vitro assays were performed to evaluate their effects on the proliferative ability mediated by NOTCH3. We identified microRNA-1299 (miR-1299) as a novel negative regulator of NOTCH3. miR-1299 was downregulated in OC and was found to be considerably correlated with tumor differentia-
\end{abstract}

Correspondence to: Professor Wei Cui, State Key Laboratory of Molecular Oncology, Department of Clinical Laboratory, National Cancer Center/National Clinical Research Center for Cancer/Cancer Hospital, Chinese Academy of Medical Sciences and Peking Union Medical College, 17 Panjiayuannanli Road, Chaoyang, Beijing 100021, P.R. China

E-mail: wendycuiwei@sina.cn

Abbreviations: OC, ovarian cancer; NOTCH3, notch receptor 3; lncRNA, long noncoding RNA; miRNA, microRNA; ceRNA, competing endogenous RNA; NICD3, NOTCH3 intracellular domain; TSS, transcriptional start site; EMT, epithelial-mesenchymal transition; TUG1, taurine upregulated gene 1

Key words: ovarian cancer, NOTCH3, miR-1299, TUG1, cell proliferation, ceRNA tion. Upregulation of miR-1299 inhibited cell proliferation, colony formation, and 5-ethynyl-2'-deoxyuridine (EdU) incorporation, as well as induced cell cycle arrest in the G0G1 phase in OC cells. Overexpression of miR-1299 in xenograft mouse models suppressed tumor growth in vivo. The lncRNA taurine upregulated gene 1 (TUG1), acting as a sponge of miR-1299, was found to upregulate NOTCH3 expression and promote cell proliferation in OC through the competing endogenous RNA mechanism. In addition, TUG1 was found to be a potential downstream target of NOTCH3, forming a miR-1299/NOTCH3/TUG1 feedback loop in the development of OC. Collectively, our findings improve the understanding of NOTCH3-mediated regulation in $\mathrm{OC}$ pathogenesis and facilitate the development of miRNA- and lncRNA-directed diagnostics and therapeutics against this disease.

\section{Introduction}

Ovarian cancer (OC) continues to be one of the most lethal malignant tumors among women worldwide. In the United States, OC accounts for only $2.5 \%$ of all malignancies among females but for $5 \%$ of cancer deaths, making it the fifth leading cause of cancer-related deaths in females $(1,2)$. In China, the mortality rate of $\mathrm{OC}$ is ranked tenth among females throughout the country (3). The low survival rates in OC are largely driven by a late-stage diagnosis and a high rate of tumor recurrence. Although most OC patients have no evidence of disease after first treatment, approximately $19 \%$ of early OC patients and 60 to $85 \%$ of advanced OC patients experience a relapse, and recurrent OC after treatment is almost incurable (4-6). Current treatment for OC is not confined merely to surgery and classical chemotherapy. Targeted therapy, such as polyADP ribose polymerase (PARP) inhibitors, as well as immunotherapy, are promising treatments (7-10). 
The Notch signaling pathway is an important system that regulates cell proliferation, differentiation, and apoptosis. Integrated genomic analyses revealed that Notch is the main signaling pathway involved in the pathophysiology of OC $(11,12)$. The expression of notch reporter 3 (NOTCH3) was found to be elevated in OC and closely correlated with the clinical stage, pathological grade, lymph node metastasis, drug-resistant recurrence, and survival rate of OC patients (13-15). NOTCH3 promotes the malignant progression of OC by enhancing the proliferation of tumor cells, maintaining their stemness, and resisting apoptosis $(16,17)$. A better understanding of the tumor-specific regulation of NOTCH3 is crucial and may contribute to targeted therapy for OC. However, limited information regarding the molecules that regulate the expression of NOTCH3 is available.

Approximately $70 \%$ of the human genome can be transcribed, while less than $2 \%$ of the genome encodes proteins, generating thousands of non-coding transcripts (ncRNAs) $(18,19)$. Among them, microRNAs (miRNAs) and long noncoding RNAs (lncRNAs) are key regulators of gene expression at the post-transcriptional level. miRNAs are single-stranded non-coding small RNAs with a length of 17-22 nt. They are destabilizers and repressors of translation of mRNAs and regulate diverse biological functions, including proliferation, migration, apoptosis, angiogenesis, and metabolic progression in various cancers, including OC (20-24). lncRNAs are ncRNAs that are longer than $200 \mathrm{nt}$. They are implicated in multiple cancers and play critical roles in tumor initiation and progression (25-28). Recent studies have revealed that lncRNAs can act as competing endogenous RNA (ceRNA) or miRNA 'sponges' to modulate crucial genes in cancer (29-32), providing a feasible way to understand the regulatory mechanisms underlying cancer pathogenesis and identify novel diagnostic biomarkers or potential therapeutic candidates.

Given the significant oncogenic role of NOTCH3 in $\mathrm{OC}$, we aimed to determine the functional significance of NOTCH3-mediated regulatory ncRNAs in OC. In this study, we found that microRNA-1299 (miR-1299) was a novel negative regulator of NOTCH3 expression in OC with clinical significance. Downregulation of miR-1299 promoted the malignant progression of OC by tumor cell proliferation. The lncRNA taurine upregulated gene 1 (TUG1) was found to function as a ceRNA, regulating NOTCH3 expression by sponging miR-1299 in OC. TUG1 was also identified as a potential downstream target of NOTCH3 and formed a miR-1299/NOTCH3/TUG1 feedback loop. Our results elucidate the regulatory mechanism underlying NOTCH3 expression in $\mathrm{OC}$ and provide potential therapeutic targets for the anticancer therapy of OC.

\section{Materials and methods}

Cell lines/cell culture. The human OC cell lines A2780, CAOV3, and SKOV3 were purchased from the National Infrastructure of Cell Line Resources in China (Beijing, China). Cells were cultured in DMEM or RPMI-1640 medium with 10\% FBS (Thermo Fisher Scientific, Inc.) and incubated at $37^{\circ} \mathrm{C}$ with $5 \%$ $\mathrm{CO}_{2}$. Toblock Notch signaling in OC cells, the $\gamma$-secretase inhibitor N-[N-(3,5-difluorophenacetyl)-1-alanyl]-S-phenylglycine t-butyl ester(DAPT,\#HY-13027; MedChem Express) dissolved in DMSO was added in the medium.

Clinical samples. Thirty-five epithelial serous OC and 16 pathologically confirmed normal ovary tissues were collected from patients (medium age 54 years; range 30-72 years and medium age 51 years; range 35-64 years, respectively) undergoing surgery at the Cancer Hospital, Chinese Academy of Medical Sciences and Peking Union Medical College (Beijing, China) from July 2018 to June 2019. All OC cases were diagnosed by pathological evaluation and had complete clinical information. All tissue samples were immediately frozen in liquid nitrogen after resection from patients and stored at $-80^{\circ} \mathrm{C}$ for RNA extraction. This study was conducted according to the ethical guidelines of the 1975 Declaration of Helsinki and approved by the Ethics Committee of Peking Union Medical College Cancer Hospital (Beijing, China) (grant no: NCC2017G-115). Written informed consent was obtained from all of the subjects.

The OC samples were divided into high and low expression of miR-1299 and NOTCH3 groups by the cut-off value, which was defined as the cohort median.

Oligonucleotide transfection, plasmid construction, and lentiviral infection. miR-1299 mimics, inhibitors, and scramble miRNA controls were purchased from GenePharma (Shanghai, China). The lentivirus-containing short hairpin RNA (shRNA) targeting TUG1 or NOTCH3, and the plasmid expressing the active intracellular domain of NOTCH3 (NICD3) were purchased from GeneChem (Shanghai, China). The wild-type and mutated 3'UTR sequence of NOTCH3 was cloned into the pmirGLO vector (Promega) to construct recombinant plasmids named pmirGLO-NOTCH3-WT and pmirGLO-NOTCH3-MUT. Sequences of lncRNA TUG1 and XIST containing the predictive binding sites of miR-1299 and containing point mutations at the site region were cloned into the pmirGLO vector and were named pmirGLO-TUG1-WT, pmirGLO-XIST-WT, and pmirGLO-TUG1-MUT. OC cells were transfected with oligonucleotides using Lipofectamine ${ }^{\circledR}$ RNAiMAX reagent (Thermo Fisher Scientific, Inc.) and plasmids using TurboFect ${ }^{\mathrm{TM}}$ transfection reagent (Thermo Fisher Scientific, Inc.) according to the manufacturer's instructions.

$R N A$ extraction and $R T-q P C R$. Total RNA was extracted from OC tissues and cultured cell lines using TRIzol reagent (Thermo Fisher Scientific, Inc.) according to the manufacturer's instructions. mRNA and lncRNA levels were quantified with RT-qPCR using the SYBR Premix Ex Taq reverse transcription PCR kit (Takara, China), and $\beta$-actin was used as an internal control. miRNA was quantified with RT-qPCR using the hairpin-it ${ }^{\mathrm{TM}}$ microRNA and U6 snRNA normalization RT-PCR quantitation kit (GenePharma). Each assay was carried out in triplicates in a Light Cycler 480 Instrument (Roche), and the relative expression of mRNA and miRNA was calculated using the $2^{-\Delta \Delta \mathrm{Cq}}$ method (33). The primers for RT-qPCR are shown in Table SI.

Cell cycle, proliferation, and colony formation assays. For cell cycle analysis, cells were collected and adjusted to $1 \times 10^{6} / \mathrm{ml}$ $48 \mathrm{~h}$ after transfection. Ethanol (70\%) was used to fix the cells, and RNase A (100 $\mu \mathrm{g} / \mathrm{ml})$ was used to remove RNAs. Finally, 
cells were stained with propidium iodide (PI) $(50 \mu \mathrm{g} / \mathrm{ml})$ at room temperature for $30 \mathrm{~min}$ and analyzed using LSRII flow cytometry (BD Biosciences). Cell viability was detected using the Cell Counting Kit-8 (CCK-8) (Dojindo, Japan) according to the manufacturer's instructions. Twenty-four hours after transfection, the cells were seeded in 96-well plates at 3,000 cells/well. The proliferative ability of cells was determined at $0,24,48,72$ and $96 \mathrm{~h}$ by measuring the absorbance values at a wavelength of $450 \mathrm{~nm}$. For colony formation assay, OC cells (800 cells per well) were seeded into 6-well plates $24 \mathrm{~h}$ after transfection. After 10 days of incubation, the cells were fixed in methanol and stained with $0.1 \%$ crystal violet. The colonies were counted using a GBOX F3 gel documentation system (Syngene, Cambridge, UK).

Immunofluorescence (IF) assay. A total of 10,000 cells were seeded on glass coverslips in 24-well plates and grown overnight. For the EdU incorporation assay, $10 \mu \mathrm{M}$ EdU was used to treat the cells, and an EdU cell proliferation kit (Sangon) was used for measurement. Cellular nuclei were stained using $5 \mu \mathrm{g} / \mathrm{ml}$ Hoechst 33342 at $26^{\circ} \mathrm{C}$ for 30 min. Images were observed under a DMI 4000 confocal laser scanning microscope at x200 magnification (Leica, Frankfurt, Germany).

Luciferase reporter assay. Cells were seeded in 96-well plates at $1.5 \times 10^{4}$ cells/well. When the cells reached $60 \%$ confluence, vectors containing the wild-type or mutant 3'UTR of NOTCH3 and wild-type or mutant binding site sequence of TUG1 were co-transfected with miR-1299 mimics and scramble miRNA using Turbofect (Thermo Fisher Scientific, Inc.). Forty-eight hours after transfection, luciferase activity was measured using the Dual-Luciferase Reporter assay system (Promega) and expressed as the ratio of firefly and Renilla luciferase activities.

Western blotting. Proteins were extracted from cells or tissues using RIPA lysis buffer (Solarbio) with protease inhibitors and phosphatase inhibitors. A total of $30 \mu \mathrm{g}$ proteins were loaded per lane, separated on 10\% SDS-PAGE gels, and blotted on polyvinylidene difluoride membrane. After being blocked with $5 \%$ skim milk for $2 \mathrm{~h}$ at room temperature, the membranes were incubated with primary antibodies (dilution 1:1,000) overnight at $4^{\circ} \mathrm{C}$. The primary antibodies used in this study were NOTCH3 antibody (product code ab23426; Abcam) and GAPDH (cat. no. 2118; Cell Signaling Technology, Inc.). Anti-mouse $\mathrm{IgG}$, peroxidase-linked antibody was used as secondary antibody (dilution 1:2,500; cat. no. 5174; Cell Signaling Technology, Inc.) and was incubated at room temperature for $1 \mathrm{~h}$. The blots were detected using a chemiluminescence kit (cat. no. 34577; Thermo Fisher Scientific, Inc.) and imaged using MiniChemi 610 system (Sage Creation Science, Co., Ltd.).

In vivo animal experiments. Eight four-week-old female $\mathrm{BALB} / \mathrm{c}$ nude mice (body weight range, 17.1-18.2 g) were purchased from Beijing Vital River Laboratory Animal Technology Co., Ltd. and randomly divided into two groups. The mice were housed with filtered air, $12 \mathrm{~h}$ light/dark cycle, constant temperature $\left(25^{\circ} \mathrm{C}\right)$ and had free access to sterilized food and water. After $24-\mathrm{h}$ transfection, $2 \times 10^{6}$ cells containing miR-1299 or NC agomir were infected into the right armpit of the mice. miR-1299 agomir or NC agomir (RiboBio) was directly injected into the implanted tumor at the dose of $2 \mathrm{nmol} / 30 \mu \mathrm{l}$ phosphate-buffered saline (PBS) per mouse every 6 days for 6 times. Tumor growth was monitored by measuring the tumor volume $(\mathrm{V})$ every 6 days with a Vernier caliper and calculated as: $V=$ length $x$ width ${ }^{2} / 2$. All the mice were anesthetized with sodium pentobarbital $(500 \mathrm{mg} / \mathrm{kg}$, intraperitoneally) and sacrificed by cervical vertebra dislocation on day 42 or when the tumor volume reached the threshold of $1,500 \mathrm{~mm}^{3}$, and tumors were weighed and snap-frozen for protein and RNA extraction.

Animal experiments were conducted with the approval of the Animal Ethics Committee of Cancer Hospital, Chinese Academy of Medical Sciences and Peking Union Medical College, Beijing (ACC2019A060) and in accordance with the Guide for the Care and Use of Laboratory Animals by the US National Institutes of Health.

Statistical analysis. SPSS software version 25.0 (IBM Corp.) and GraphPad Prism 8 (GraphPad Software, Inc.) were used for statistical analysis. Each experiment was performed at least in triplicate, and numerical data are expressed as means \pm SD. The difference in clinicopathological features between two groups was determined by independent-sample Student's t-test or Mann-Whitney U test for continuous variables and the Chi-square test or Fisher exact test for categorical variables. One-way ANOVA analysis with Tukey post hoc test was used for comparisons among multiple groups. Pearson's correlation analysis was used to examine the relationship between two gene expression levels. A value of $\mathrm{P}<0.05$ was indicative of statistical significance.

\section{Results}

miR-1299 is a negative regulator of NOTCH3 in OC with clinical significance. To investigate the potential miRNAs that regulate NOTCH3, we firstly performed a bioinformatics analysis using the miRWalk database (34), which integrated predicted gene-miRNA target information from 13 databases and compared the results with miRNAs reported to be significantly downregulated in previously published miRNA profiles (NCBI/GEO/GSE47841) (35) (Table SII). The two screening methods overlapped on only 11 miRNAs among which miR-1299 had the highest score in miRWalk. Therefore, we focused our subsequent analysis on miR-1299 as a putative regulator of NOTCH3 in OC. We measured the level of mature miR-1299 and NOTCH3 in 35 fresh OC tissues and 16 normal ovarian tissues, as well as in four OC cell lines. miR-1299 expression was significantly downregulated in the OC tissues compared with that in the normal tissue $(\mathrm{P}<0.05$, Fig. 1A). There was an inverse correlation between the level of miR-1299 and NOTCH3 both in OC tissues (Fig. 1B) and in OC cell lines (Fig. 1C).

To confirm the regulation of miR-1299 on NOTCH3 expression, we first transfected miR-1299 mimics and NC scramble into miR-1299 low-expressing cell A2780 and CAOV3. RT-qPCR results showed that NOTCH3 and its pathway genes were largely reduced in the A2780 
A

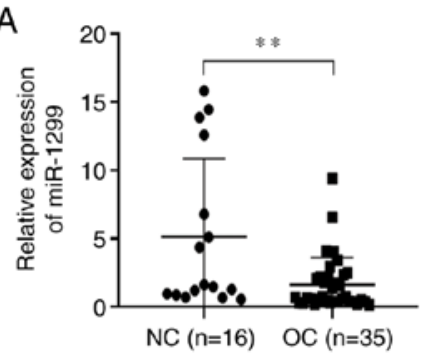

B

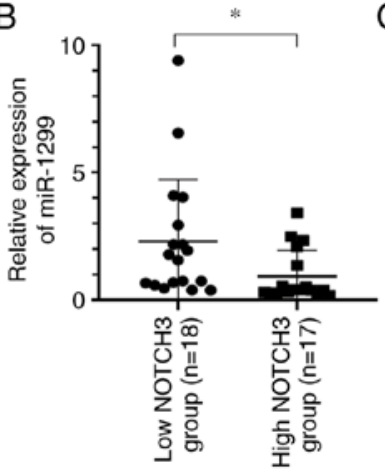

D

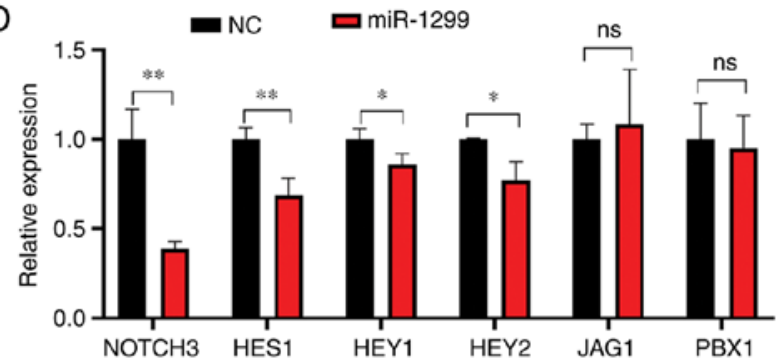

$\mathrm{F}$

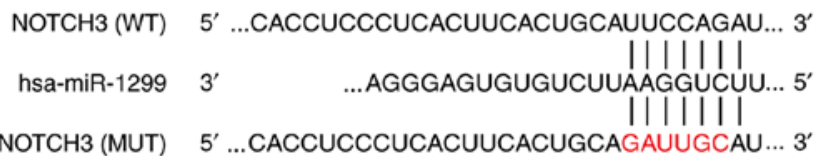

C

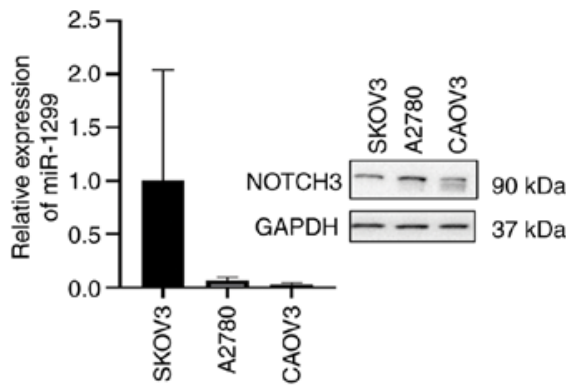

E

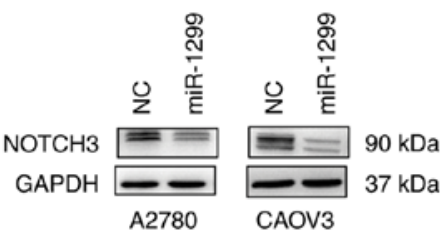

G

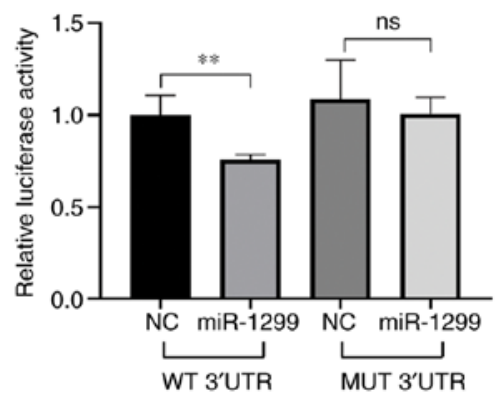

Figure 1. miR-1299 is downregulated in OC and acts as a negative regulator of NOTCH3. (A) Analysis of miR-1299 expression in OC and normal ovary tissues (NC) using RT-qPCR. (B) miR-1299 expression was significantly lower in OC tissues with high NOTCH3 expression compared to those with low NOTCH3 expression. (C) Correlation between miR-1299 and NOTCH3 levels in OC cell lines. (D) RT-qPCR analysis of NOTCH3 pathway genes after transfection of miR-1299 mimics in A2780 cells. (E) Western blot analysis of NOTCH3 after transfection of miR-1299 mimics in A2780 and CAOV3 cell lines. (F) Putative miR-1299 binding sequence in NOTCH3 3'UTR (untranslated region) (WT) and the mutated 3'UTR sequence (MUT). (G) Relative luciferase activity of reporter plasmids carrying wild-type (WT) or mutant (MUT) NOTCH3 3'UTR in A2780 cells co-transfected with NC or miR-1299 mimics. Means \pm SD are shown. Statistical analysis was conducted using Student's t-test. " $\mathrm{P}<0.05$ and ${ }^{* *} \mathrm{P}<0.01$; ns, not significant. OC, ovarian cancer; NOTCH3, notch receptor 3; HES1, hairy and enhancer of split-1; HEY1, Hes related family BHLH transcription factor with YRPW motif 1; HEY 2, Hes related family BHLH transcription factor with YRPW motif 2; JAG1, Jagged canonical Notch ligand 1; PBX1, Pre-B-cell leukemia transcription factor 1.

(Fig. 1D) and CAOV3 cells (Fig. S1) when transfected with miR-1299 mimics, and western blotting results confirmed the decreased protein level of NOTCH3 (Fig. 1E). In turn, when we knocked down NOTCH3 or overexpressed NICD3 in OC cells, we observed a subsequent increase or decrease in the miR-1299 level (Fig. S2). Furthermore, we cloned wild-type 3'UTR sequence of NOTCH3 and 3'UTR containing point mutations in the putative binding sites into a luciferase reporter plasmid (pmirGLO-NOTCH3-WT and pmirGLO-NOTCH3-MUT) (Fig. 1F). Both plasmids were co-transfected with miR-1299 mimics or NC mimics separately in A2780 cells. The results showed that overexpression of miR-1299 suppressed luciferase activity significantly in cells transfected with pmirGLO-NOTCH3-WT plasmid but not in those transfected with pmirGLO-NOTCH3-mutant vectors (Fig. 1G). These observations suggested that miR-1299 bound directly to the predicted binding sites in the NOTCH3 3'UTR region and negatively regulated NOTCH3 expression.
The association of miR-1299 level and clinicopathological features was also analyzed in OC patients (Table I). Low expression of miR-1299 was significantly correlated with low tumor differentiation. Decreased miR-1299 level was associated with younger age, advanced tumor stage, and necessity to receive neoadjuvant chemotherapy before surgery; however, the association was not statistically significant because of the limited number of cases. Thus, we considered miR-1299 as a regulator of NOTCH3 and hypothesized that decreased expression of miR-1299 promoted OC progression and development.

miR-1299 inhibits OC cell proliferation, colony formation, and cell cycle in vitro. Subsequently, we investigated the role of miR-1299 in OC by transfecting miR-1299 mimics in OC cell lines A2780 and CAOV3 (Fig. 2A), both of which had an endogenous low miR-1299 expression. Overexpression of miR-1299 in OC cells significantly reduced cell proliferation, as observed with a CCK- 8 assay compared to cells transfected with scrambled miRNA (NC mimic) (Fig. 2B). Colony forma- 
Table I. Association between miR-1299 levels and clinicopathological characteristics of the OC patients.

miR-1299 expression

\begin{tabular}{|c|c|c|c|}
\hline \multirow[b]{2}{*}{ Characteristics } & & \multirow[b]{2}{*}{ P-value } \\
\hline & High expression $(\mathrm{n}=17)$ & Low expression $(\mathrm{n}=18)$ & \\
\hline Mean age (years) & $55.2 \pm 2.8$ & $52.9 \pm 1.8$ & 0.121 \\
\hline \multicolumn{4}{|l|}{ Tumor stage } \\
\hline I-II & 3 & 0 & 0.057 \\
\hline III & 13 & 13 & \\
\hline IV & 1 & 5 & \\
\hline Tumor differentiation & & & 0.045 \\
\hline High/median & 4 & 0 & \\
\hline Low & 13 & 18 & \\
\hline Nodal metastasis & & & 0.305 \\
\hline No & 12 & 9 & \\
\hline Yes & 5 & 9 & \\
\hline Neoadjuvant chemotherapy & & & 0.060 \\
\hline No & 15 & 10 & \\
\hline Yes & 2 & 8 & \\
\hline Serum CA125 level at initial diagnosis & $534.1(108.0,1,362.0)$ & $1017.3(197.4,1,676.0)$ & 0.211 \\
\hline
\end{tabular}

tion assay showed that treatment with miR-1299 mimics decreased the number of colonies formed by OC cell lines (Fig. 2C). EdU incorporation assay revealed that miR-1299 inhibited DNA synthesis in cell proliferation (Fig. 2D). Additionally, cell cycle analysis showed that transfection of miR-1299 mimics significantly blocked the cells in the G0/G1 phase, accompanied by a smaller population in $\mathrm{S}$ and $\mathrm{G} 2$ phases (Fig. 2E).

miR-1299 suppresses tumor growth in vivo. We further examined the impact of miR-1299 on OC in a xenograft nude mouse model. A2780 cells transfected with miR-1299 or NC mimics were implanted subcutaneously in BALB/c nude mice. Starting on day 8 post-implantation, miR-1299 or NC mimics were injected intratumorally every 6 days for 6 times. In vivo tumor growth was evaluated by measuring tumor volume and final weight. We observed that treatment with miR-1299 mimics significantly inhibited tumor growth in vivo. The tumor volume and weight were significantly lower in the miR-1299 mimic group than in the NC group (Fig. 3A-C), substantiating the tumor suppressor function of miR-1299 in OC tumorigenesis. Moreover, decreased NOTCH3 protein level in the xenograft tissues with overexpression of miR-1299 was further confirmed (Fig. 3D).

lncRNA TUG1 functions as a sponge of miR-1299 and promotes cell proliferation in $O C$. To investigate the potential regulators of NOTCH3 by ceRNA mechanism, we first performed a comprehensive bioinformatics analysis using predicted miR-1299-targeted lncRNAs from DIANA databases (36), and OC-relevant IncRNAs from Starbase database (37) and lncRNA disease database (38) (Fig. 4A). The three-screening data overlapped on only two lncRNAs, TUG1 and XIST (X-inactive specific transcript). Therefore, we constructed luciferase reporter plasmids containing the two highest scoring putative miR-1299 binding sites in TUG1 (pmirGLO-TUG1-WT1 and pmirGLO-TUG1-WT2) and XIST (pmirGLO-XIST-WT1 and pmirGLO-XIST-WT2). After co-transfecting the plasmids with miR-1299 mimics or NC mimics in A2780, we found that overexpression of miR-1299 suppressed luciferase activity significantly in two wild-type plasmids of TUG1 (Fig. 4B and C) but not in those of XIST (Fig. S3). We also constructed plasmids carrying point mutations in either binding site of TUG1 (pmirGLO-TUG1-MUT1 and pmirGLO-TUG1-MUT2), and no significant changes in luciferase activity was observed in cells transfected with the two mutant plasmids (Fig. 4B and C). RT-qPCR further revealed that miR-1299 could be upregulated in OC cells after knockdown of TUG1 by lentiviral shRNA particles (Fig. 4D and E). Taken together, these results indicated that the IncRNA TUG1 was a direct sponge of miR-1299 in OC.

We next evaluated the potential tumorigenicity of TUG1 in OC. As expected, downregulation of TUG1 by shRNA resulted in the inhibition of OC cells in terms of cell proliferation, colony formation, EdU incorporation, and cell cycle, which could be partially rescued by inhibition of miR-1299 (Fig. 4F-I). Moreover, knockdown of TUG1 reduced NOTCH3 expression in OC cells, and miR-1299 inhibitors partially rescued the NOTCH3 level (Fig. 4J). This suggested that TUG1, acting as a ceRNA, affects NOTCH3 expression and promotes cell proliferation by sponging miR-1299. 

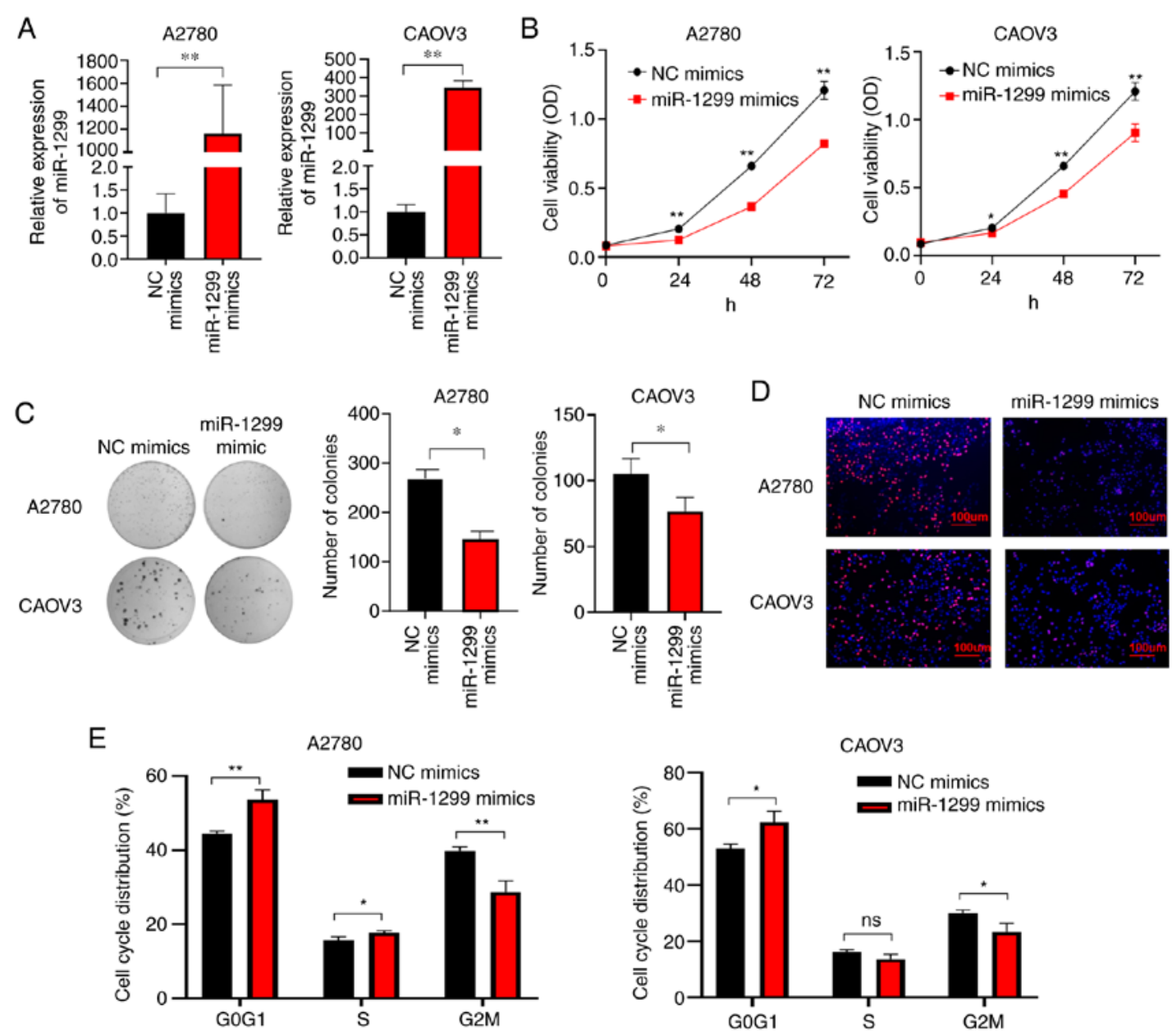

Figure 2. Upregulation of miR-1299 inhibits cell proliferation, colony formation, EdU incorporation, and cell cycle in OC A2780 and CAOV3 cell lines. (A) RT-qPCR analysis of miR-1299 expression level in OC cells after transfection of NC or miR-1299 mimics for $48 \mathrm{~h}$. (B) Cell proliferation of OC cells after transfection of NC or miR-1299 mimics by CCK-8 assay. (C) Representative photographs and quantifications of the colony formation assay after transfection of NC or miR-1299 mimics. (D) Representative photographs of EdU incorporation assay in OC cells after transfection of NC or miR-1299 mimics. (E) Cell cycle distribution of OC cells after transfection of NC or miR-1299 mimics by flow cytometry. Means \pm SD are shown. Statistical analysis was conducted using Student's t-test. ${ }^{*} \mathrm{P}<0.05$ and ${ }^{* *} \mathrm{P}<0.01$, miR-1299 mimic compared with the NC mimic; ns, not significant; OC, ovarian cancer.
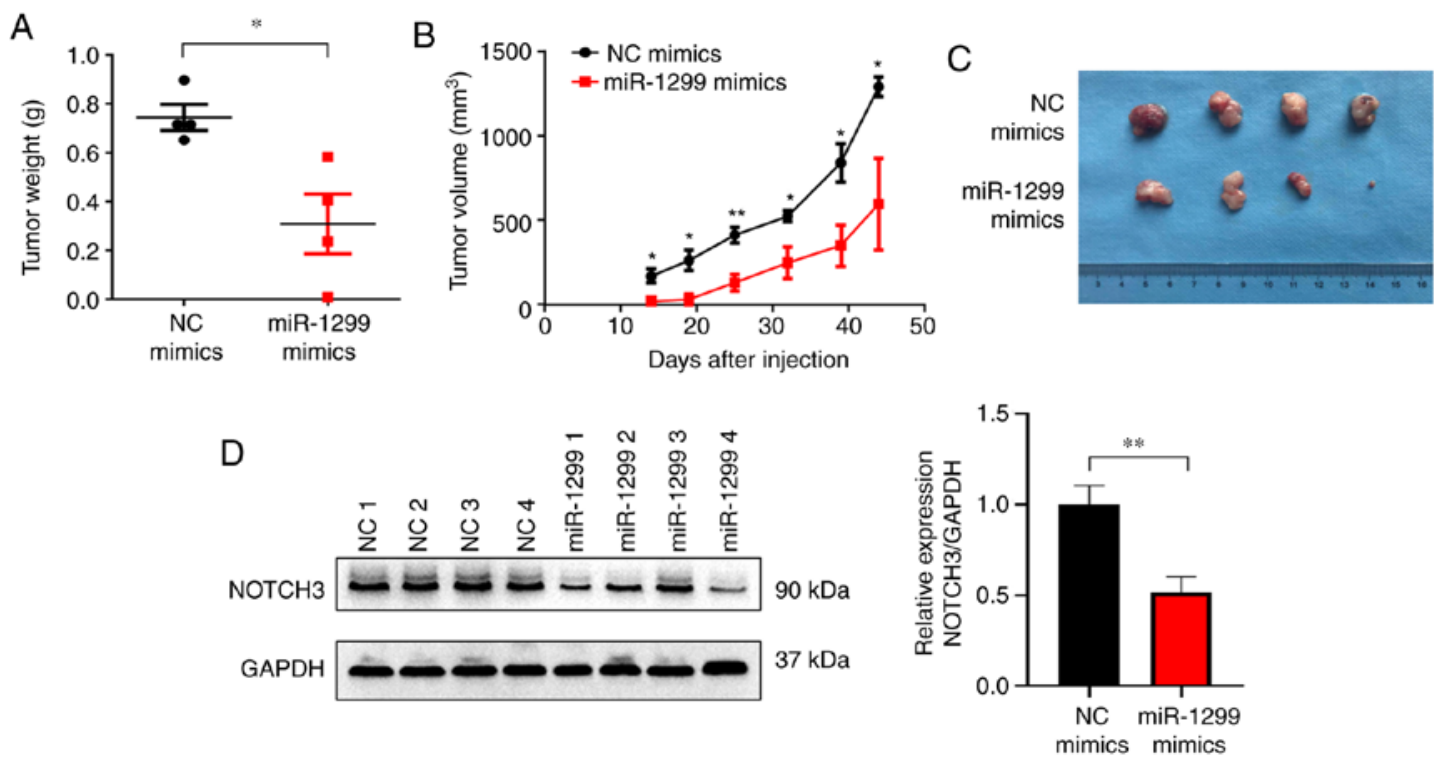

Figure 3. Ectopic miR-1299 suppresses OC tumor growth in vivo. (A) Tumor weight and (B) tumor volume in the xenograft mouse model. (C) Photographs of dissected tumors treated with NC or miR-1299 mimics. (D) Quantification of NOTCH3 protein in tumors treated with NC or miR-1299 mimics by western blotting. Means \pm SD are shown. Statistical analysis was conducted using Student's t-test. ${ }^{*} \mathrm{P}<0.05$ and ${ }^{* *} \mathrm{P}<0.01$, miR-1299 mimic compared with the NC mimic. OC, ovarian cancer; NOTCH3, notch receptor 3. 
A

216 miR-1299-IncRNAs 5068 OC-related IncRNAs predicted by DIANA predicted by Starbase

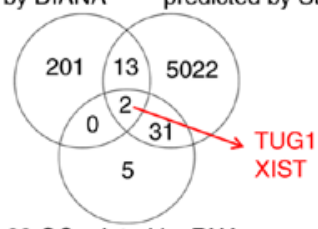

38 OC related IncRNAs predicted by LncACTdb
B

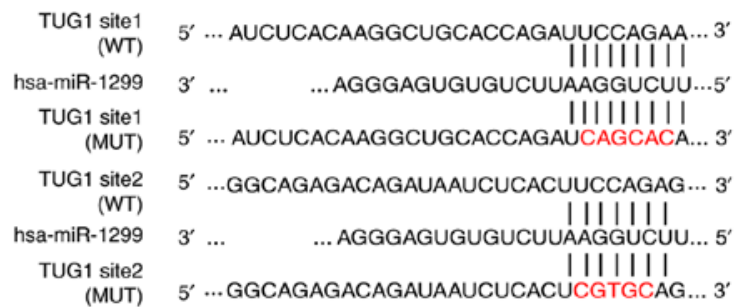

C

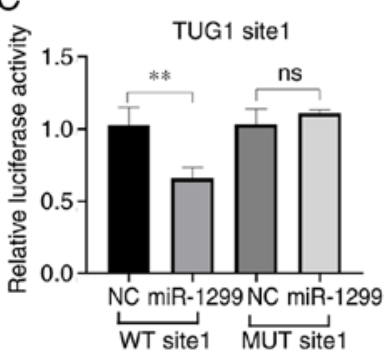

F

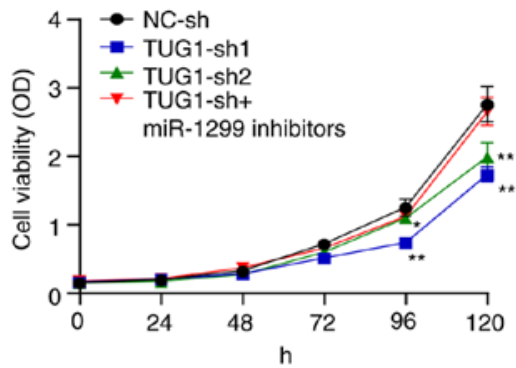

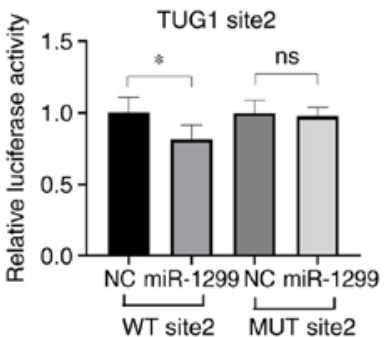

G

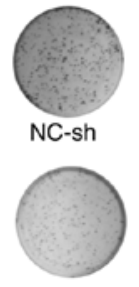

TUG1-sh2
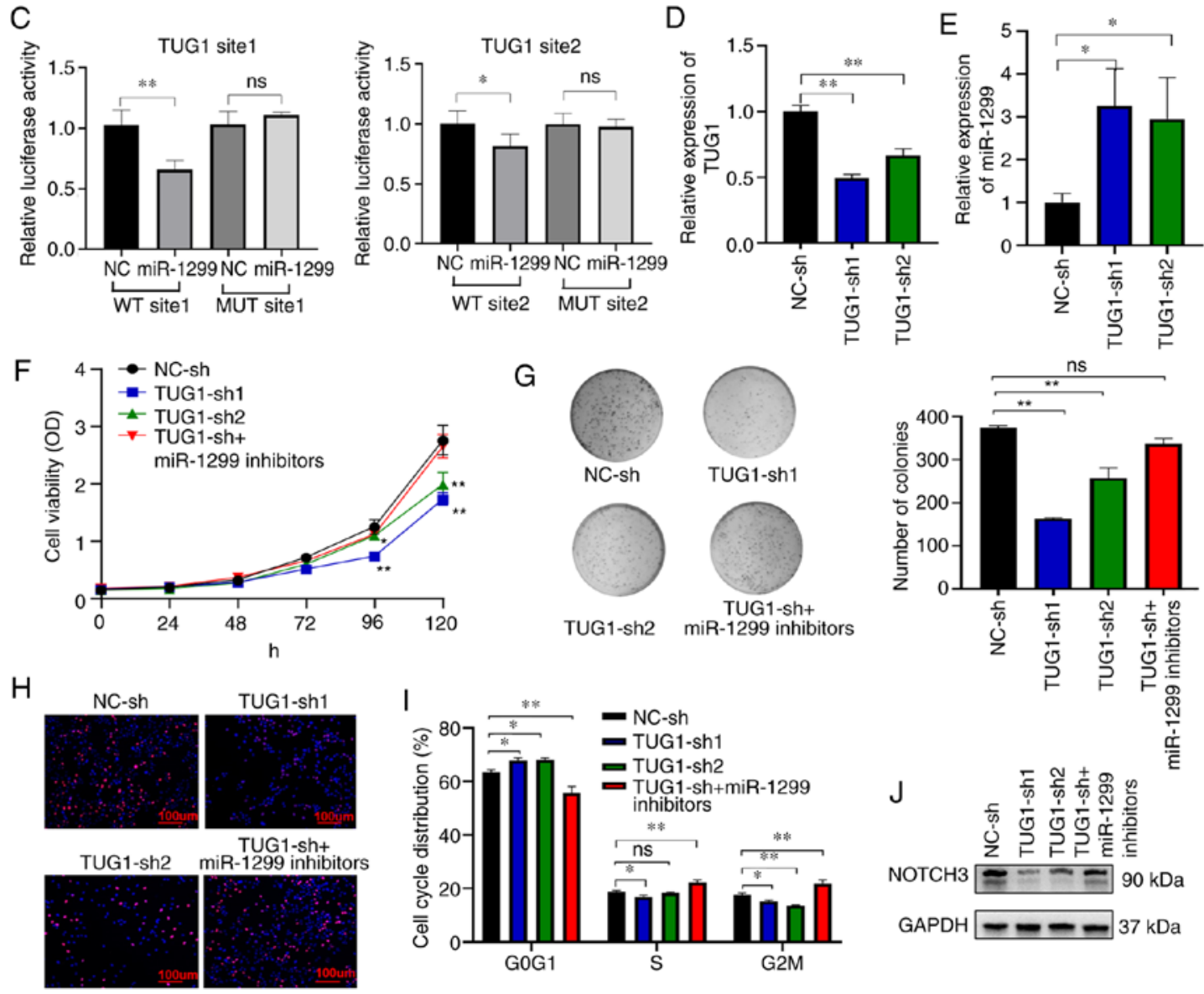

$J$

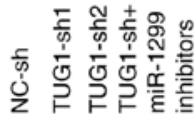

$\mathrm{NOTCH} 3=--\infty 90 \mathrm{kDa}$

GAPDH $-\longrightarrow-037 \mathrm{kDa}$

Figure 4. IncRNA TUG1 functions as a sponge of miR-1299 and promotes cell proliferation in OC. (A) Screening methods for regulatory lncRNAs of miR-1299 in OC. (B) The two highest scoring putative miR-1299 binding sites in the TUG1 sequence (WT) and the point mutations in either binding site (MUT). (C) Relative luciferase activity of reporter plasmids carrying wild-type (WT) or mutant (MUT) TUG1 binding sites in A2780 cells co-transfected with NC or miR-1299 mimics. (D) Confirmation of TUG1 knockdown in A2780 cells transfected with TUG1 shRNA by RT-qPCR analysis. (E) RT-qPCR analysis of miR-1299 level in A2780 cells transfected with TUG1 shRNA or scrambled shRNA. Results of the (F) cell proliferation, (G) colony formation, (H) EdU incorporation assays, and (I) cell cycle analysis of A2780 cells transfected with NC-sh, TUG1-sh1, TUG1-sh2, and TUG1-sh+miR-1299 inhibitors. (J) Representative blot image of NOTCH3 protein level in A2780 cells transfected with NC-sh, TUG1-sh1, TUG1-sh2, and TUG1-sh+miR-1299 inhibitors by western blotting. Means \pm SD are shown. Statistical analysis was conducted using Student's t-test and one-way ANOVA with Tukey post hoc test. "P<0.05 and ${ }^{* *} \mathrm{P}<0.01$; ns, not significant; OC, ovarian cancer; $\mathrm{NOTCH} 3$, notch receptor 3; TUG1, lncRNA taurine upregulated gene 1.

TUG1 is a potential target of NOTCH3 and forms a miR-1299/NOTCH3/TUG1 feedback loop. In the NOTCH signaling pathway, after the cleavage of $\gamma$-secretase, the intracellular domain of NOTCH3 protein (NICD3) is translocated to the nucleus and binds the transcription factor complex RBP $\mathrm{J}_{\kappa}$ (39). When we analyzed the transcriptional start site (TSS, $-2 \mathrm{~kb}$ to $+1 \mathrm{~kb}$ ) of TUG1 in the JASPAR CORE database (40), we found 5 RBP J $\kappa$ binding motifs around its TSS (Fig. 5A), indicating that TUG1 may be directly regulated by NOTCH3. Additionally, there was a significant positive correlation between the expression of TUG1 and NOTCH3 both in our tissues and in the TCGA database (41) (Fig. 5B and C). When we treated A2780 cells with
DAPT, an inhibitor of $\gamma$-secretase, that can block the NOTCH signaling pathway without interference in NOTCH3 mRNA activity, we found a subsequent decrease in TUG1 expression in a concentration gradient manner (Fig. 5D). Therefore, we considered TUG1 as a potential downstream target of NOTCH3, and that there may exist a miR-1299/NOTCH3/TUG1 feedback loop in the development of OC (Fig. 5E).

\section{Discussion}

Recently, miR-1299 was found to be downregulated in several types of cancer and to act as a tumor suppressor. Zhu et al (42) 

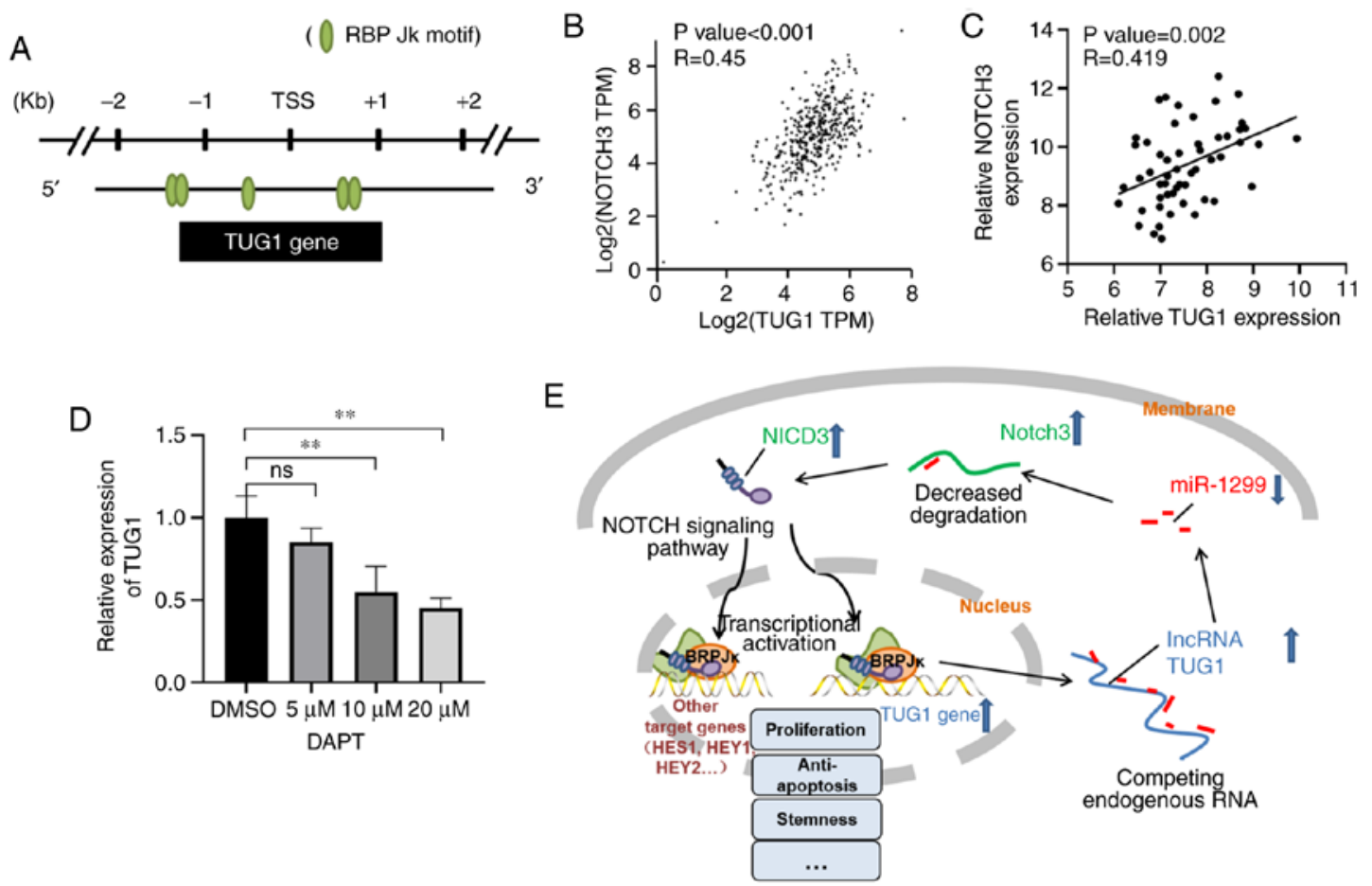

Figure 5. TUG1 is a potential downstream target of NOTCH3. (A) Schematic diagram showing RBP Jא motifs around the transcriptional start site (TSS) of the TUG1 gene. (B and C) TUG1 expression was inversely correlated with NOTCH3 mRNA in fresh OC tissues (B) and in the TCGA database (C). (D) RT-qPCR analysis of TUG1 level in A2780 cells treated with DAPT. (E) Diagrams showing the miR-1299/NOTCH3/TUG1 feedback loop in the development of OC. Statistical analysis was conducted using one-way ANOVA with Tukey post hoc test and Pearson's correlation analysis. ${ }^{* *} \mathrm{P}<0.01$; ns, not significant; OC, ovarian cancer; NOTCH3, notch receptor 3; TUG1, IncRNA taurine upregulated gene 1; NICD3, NOTCH3 intracellular domain; HES1, hairy and enhancer of split-1; HEY1, Hes related family BHLH transcription factor with YRPW motif 1; HEY 2, Hes related family BHLH transcription factor with YRPW motif 2.

showed that in hepatocellular cancer, miR-1299 overexpression inhibited cell proliferation and arrested the cell cycle in the G0/G1 phase, while miR-1299 knockdown promoted cell proliferation and accelerated G1/S transition. In breast cancer, miR-1299 was found to inhibit CDK6 expression and to bind to its 3 'UTR region, suppressing cell proliferation and migration ability (43). A similar tumor suppressive role of miR-1299 was found in esophageal squamous cell carcinoma (44), prostate cancer (45), triple-negative breast cancer (46), and cholangiocarcinoma (47). In this study, we demonstrated that miR-1299 was significantly decreased in OC tissues and was associated with clinical features, and overexpression of miR-1299 inhibited tumor growth both in vitro and in vivo, suggesting that miR-1299 also acted as a tumor suppressor in ovarian cancer (OC). In addition, we first demonstrated that miR-1299 was a negative regulator of NOTCH3, a vital oncogene in OC. Transcriptome analysis showed that suppression of NOTCH signaling in ovarian and breast cancer cells led to downregulation of genes in pathways involved in cell-cycle regulation (48). Both NOTCH3 siRNA and pathway inhibitors caused cell cycle arrest by reducing cyclin D1 and cyclin D3 levels while elevating p21 and p27 levels. In this study, we observed a similar cellular phenotype and protein change (data not shown) after transfection of miR-1299. However, there is also a limitation to our study as we used miR-1299 mimics to transfect two cell lines, and did not use inhibitors in the functional experiments considering the low expression of miR-1299 in OC.

The IncRNA taurine upregulated gene 1 (TUG1), a 7.1-kb IncRNA, was first discovered in a genomic screen for genes upregulated by taurine treatment in mouse retinal cells (49).
Recently, TUG1 was characterized as a new oncogene and found to be upregulated in multiple human cancers (50-56). TUG1 was upregulated in OC tissues and cells and was positively correlated with advanced disease and poor prognosis. Knockdown of TUG1 significantly inhibited cell proliferation and epithelial-mesenchymal transition (EMT) and induced cell apoptosis in OC (56-58). The biological functions of IncRNAs largely rely on their distinct subcellular localization. Cytoplasmic lncRNAs can regulate mRNA stability or translation by acting as sponges for miRNAs (59). TUG1 localizes both in the nucleus and the cytoplasm, indicating that it could have a multi-oncogenic role. Using bioinformatics analyses and luciferase reporter assays, our study provided the first evidence that TUG1 directly bound to and inhibited miR-1299 expression. Knockdown of TUG1 was found to inhibit cell proliferation, colony formation, and cell cycle progression, which could be partially rescued by inhibition of miR-1299. Our observations were consistent with previous studies and confirmed the involvement of TUG1 in the ceRNA mechanism. Notably, there have been several other miRNAs negatively regulated by TUG1 in cancers (60-62), among which quite a few miRNAs have been reported to have a tumor suppressive role in OC (63-65). TUG1 could sponge more than one miRNA in OC and affect the development of OC through different signaling pathways.

Interestingly, we found that TUG1 possessed five RBP Jk motifs around its promoter region, which can be bound by the NOTCH intracellular domain and trigger transcriptional activation by classical NOTCH signaling pathway. Additionally, treatment with DAPT, an inhibitor of $\gamma$-secretase, in OC cells decreased the TUG1 level in a concentration gradient-depen- 
dent manner. Therefore, we hypothesized that there could be a miR-1299/NOTCH3/TUG1 feedback loop in OC development. Downregulation of miR-1299 in OC upregulated NOTCH3 level, and overexpression of NOTCH3 activated the expression of various oncogenes by the NOTCH signaling pathway, including the downstream molecule TUG1. In addition, overexpression of TUG1 was a sponge for miR-1299, triggering a positive feedback in tumorigenesis. A recent study in glioma showed that TUG1 was a NOTCH-regulated lncRNA (66), which confirmed our hypothesis. However, we have provided preliminary evidence on this feedback loop hypothesis. The direct regulation of TUG1 by NOTCH3 at the molecular level requires further experimental evidence such as RIP or RNA pulldown results. Therefore, further confirmation of the miR-1299/NOTCH3/TUG1 feedback loop, as well as its role in other important issues, including stemness and drug-resistance in $\mathrm{OC}$, will be the main aim of our future work.

To date, the possible regulators and mechanisms underlying NOTCH3-mediated regulatory behaviors in OC have not been completely elucidated. Our study showed that miR-1299 is a novel negative regulator of NOTCH3 and is downregulated in OC. Overexpression of miR-1299 was found to play a tumor suppressor role both in vitro and in vivo by partially inhibiting cell proliferation. 1ncRNA TUG1 acted as a sponge for miR-1299 and promoted cell proliferation by upregulating NOTCH3. TUG1 was also a potential target of NOTCH3, forming a miR-1299/NOTCH3/TUG1 feedback loop in OC cells. Our findings improve the understanding of OC pathogenesis and facilitate the development of miRNAand lncRNA-targeted diagnostics and therapeutics against this disease.

\section{Acknowledgement}

Not applicable.

\section{Funding}

This work was supported by grants from the National Natural Science Foundation of China (grant no. 81772272) and the Graduate Innovation Fund of Peking Union Medical College (grant no. 2018-1002-01-27).

\section{Availability of data and materials}

The datasets used and/or analyzed during the current study are available from the corresponding author on reasonable request.

\section{Authors' contributions}

YP, DZ and WC designed the research and analyzed the data. YP, KL and XL performed the cell function experiments. YP and $\mathrm{XD}$ performed the animal experiments. YW, WW and NL collected the OC and normal ovary tissues and reviewed the clinical features. YP prepared the images and drafted the original manuscript. WC and DZ reviewed and edited the manuscript. All authors read and approved the manuscript and agree to be accountable for all aspects of the research in ensuring that the accuracy or integrity of any part of the work are appropriately investigated and resolved.

\section{Ethics approval and consent to participate}

The present study was approved by the Research Ethics Committee of the Cancer Hospital, Chinese Academy of Medical Sciences and Peking Union Medical College, Beijing [grant no: NCC2017G-115], and informed consent was obtained from all participating patients. Animal experiments were conducted with the approval of the Animal Ethics Committee of Cancer Hospital, Chinese Academy of Medical Sciences and Peking Union Medical College, Beijing (ACC2019A060) and in accordance with the Guide for the Care and Use of Laboratory Animals by the US National Institutes of Health.

\section{Patient consent for publication}

Not applicable.

\section{Competing interests}

The authors declare that they have no competing interests.

\section{References}

1. Torre LA, Trabert B, DeSantis CE, Miller KD, Samimi G, Runowicz CD, Gaudet MM, Jemal A and Siegel RL: Ovarian cancer statistics, 2018. CA Cancer J Clin 68: 284-296, 2018.

2. Siegel RL, Miller KD and Jemal A: Cancer statistics, 2019. CA Cancer J Clin 69: 7-34, 2019.

3. Zheng RS, Sun KX, Zhang SW, Zeng HM, Zou XN, Chen R, $\mathrm{Gu}$ XY, Wei WW and He J: Report of cancer epidemiology in China, 2015. Zhonghua Zhong Liu Za Zhi 41: 19-28, 2019 (In Chinese).

4. Marchetti C, Palaia I, De Felice F, Musella A, Donfracesco C, Vertechy L, Romito A, Piacenti I, Musio D, Muzii L, et al: Tyrosine-kinases inhibitors in recurrent platinum-resistant ovarian cancer patients. Cancer Treat Rev 42: 41-46, 2016.

5. du Bois A, Reuss A, Pujade-Lauraine E, Harter P, Ray-Coquard I and Pfisterer J: Role of surgical outcome as prognostic factor in advanced epithelial ovarian cancer: A combined exploratory analysis of 3 prospectively randomized phase 3 multicenter trials: By the Arbeitsgemeinschaft Gynaekologische Onkologie Studiengruppe Ovarialkarzinom (AGO-OVAR) and the Groupe d'Investigateurs Nationaux Pour les Etudes des Cancers de l'Ovaire (GINECO). Cancer 115: 1234-1244, 2009.

6. Zang RY, Harter P, Chi DS, Sehouli J, Jiang R, Tropé CG, Ayhan A, Cormio G, Xing Y, Wollschlaeger KM, et al: Predictors of survival in patients with recurrent ovarian cancer undergoing secondary cytoreductive surgery based on the pooled analysis of an international collaborative cohort. Br J Cancer 105: 890-896, 2011.

7. Ottevanger PB: Ovarian cancer stem cells more questions than answers. Semin Cancer Biol 44: 67-71, 2017.

8. Mirza MR, Monk BJ, Herrstedt J, Oza AM, Mahner S, Redondo A, Fabbro M, Ledermann JA, Lorusso D, Vergote I, et al: Niraparib maintenance therapy in platinum-sensitive, recurrent ovarian cancer. N Engl J Med 375: 2154-2164, 2016.

9. Lee EK and Konstantinopoulos PA: Combined PARP and immune checkpoint inhibition in ovarian cancer. Trends Cancer 5: 524-528, 2019.

10. Mitsuhashi Y, Horiuchi A, Miyamoto T, Kashima H, Suzuki A and Shiozawa T: Prognostic significance of Notch signalling molecules and their involvement in the invasiveness of endometrial carcinoma cells. Histopathology 60: 826-837, 2012.

11. Cancer Genome Atlas Research Network: Integrated genomic analyses of ovarian carcinoma. Nature 474: 609-615, 2011.

12. Park JT, Li M, Nakayama K, Mao TL, Davidson B, Zhang Z, Kurman RJ, Eberhart CG, Shih IeM and Wang TL: Notch3 gene amplification in ovarian cancer. Cancer Res 66: 6312-6318, 2006.

13. Liu Z, Yun R, Yu X, Hu H, Huang G, Tan B and Chen T: Overexpression of Notch 3 and pS6 is associated with poor prognosis in human ovarian epithelial cancer. Mediators Inflamm 2016: 5953498, 2016. 
14. Jung SG, Kwon YD, Song JA, Back MJ,Lee SY,Lee C, Hwang YY and An HJ: Prognostic significance of Notch 3 gene expression in ovarian serous carcinoma. Cancer Sci 101: 1977-1983, 2010.

15. Park JT, Chen X, Tropè CG, Davidson B, Shih IeM and Wang TL: Notch3 overexpression is related to the recurrence of ovarian cancer and confers resistance to carboplatin. Am J Pathol 177: 1087-1094, 2010.

16. Hu W, Liu T, Ivan C, Sun Y, Huang J, Mangala LS, Miyake T, Dalton HJ, Pradeep S, Rupaimoole R, et al: Notch3 pathway alterations in ovarian cancer. Cancer Res 74: 3282-3293, 2014.

17. Gupta N, Xu Z, El-Sehemy A, Steed H and Fu Y: Notch3 induces epithelial-mesenchymal transition and attenuates carboplatin-induced apoptosis in ovarian cancer cells. Gynecol Oncol 130: 200-206, 2013.

18. Djebali S, Davis CA, Merkel A, Dobin A, Lassmann T,Mortazavi A, Tanzer A, Lagarde J, Lin W, Schlesinger F, et al: Landscape of transcription in human cells. Nature 489: 101-108, 2012.

19. Derrien T, Guigó R and Johnson R: The long non-coding RNAs: A new (P)layer in the 'Dark Matter'. Front Genet 2: 107, 2012.

20. Yang Y, Ishak Gabra MB, Hanse EA, Lowman XH, Tran TQ Li H, Milman N, Liu J, Reid MA, Locasale JW, et al: MiR-135 suppresses glycolysis and promotes pancreatic cancer cell adaptation to metabolic stress by targeting phosphofructokinase-1. Nat Commun 10: 809, 2019.

21. Tang W, Zhou W, Xiang L, Wu X, Zhang P, Wang J, Liu G, Zhang W, Peng Y, Huang X, et al: The p300/YY1/miR-500a-5p/ HDAC2 signalling axis regulates cell proliferation in human colorectal cancer. Nat Commun 10: 663, 2019.

22. Huang T, Wan X, Alvarez AA, James CD, Song X, Yang Y, Sastry N, Nakano I, Sulman EP, Hu B and Cheng SY: MIR93 (microRNA-93) regulates tumorigenicity and therapy response of glioblastoma by targeting autophagy. Autophagy 15: 1100-1111, 2019.

23. Zhang Z, Zhang L, Wang B, Wei R, Wang Y, Wan J, Zhang C, Zhao L, Zhu X, Zhang Y, et al: MiR-337-3p suppresses proliferation of epithelial ovarian cancer by targeting PIK3CA and PIK3CB. Cancer Lett 469: 54-67, 2020.

24. Tung CH, Kuo LW, Huang MF, Wu YY, Tsai YT, Wu JE, Hsu KF Chen YL and Hong TM: MicroRNA-150-5p promotes cell motility by inhibiting c-Myb-mediated Slug suppression and is a prognostic biomarker for recurrent ovarian cancer. Oncogene 39 862-876, 2020.

25. Lin $\mathrm{C}$ and Yang L: Long noncoding RNA in cancer: Wiring signaling circuitry. Trends Cell Biol 28: 287-301, 2018.

26. Klingenberg M, Matsuda A,Diederichs S and Patel T: Non-coding RNA in hepatocellular carcinoma: Mechanisms, biomarkers and therapeutic targets. J Hepatol 67: 603-618, 2017.

27. Schmitt AM and Chang HY: Long noncoding RNAs in cancer pathways. Cancer Cell 29: 452-463, 2016.

28. Huarte M: The emerging role of lncRNAs in cancer. Nat Med 21: $1253-1261,2015$

29. Wang CJ, Zhu CC, Xu J, Wang M, Zhao WY, Liu Q, Zhao G and Zhang ZZ: The lncRNA UCA1 promotes proliferation, migration, immune escape and inhibits apoptosis in gastric cancer by sponging anti-tumor miRNAs. Mol Cancer 18: 115, 2019.

30. Lu W, Zhang H, Niu Y, Wu Y, Sun W, Li H, Kong J, Ding K, Shen $\mathrm{HM}, \mathrm{Wu} \mathrm{H}$, et al: Long non-coding RNA linc00673 regulated non-small cell lung cancer proliferation, migration, invasion and epithelial mesenchymal transition by sponging miR-150-5p. Mol Cancer 16: 118, 2017.

31. Du Z, Sun T, Hacisuleyman E, Fei T, Wang X, Brown M, Rinn JL, Lee MG, Chen Y, Kantoff PW and Liu XS: Integrative analyses reveal a long noncoding RNA-mediated sponge regulatory network in prostate cancer. Nat Commun 7: 10982, 2016.

32. Zheng ZQ, Li ZX, Zhou GQ, Lin L, Zhang LL, Lv JW, Huang XD, Liu RQ, Chen F, He XJ, et al: Long noncoding RNA FAM225A promotes nasopharyngeal carcinoma tumorigenesis and metastasis by acting as ceRNA to sponge miR-590-3p/miR-1275 and upregulate ITGB3. Cancer Res 79: 4612-4626, 2019.

33. Livak KJ and Schmittgen TD: Analysis of relative gene expression data using real-time quantitative PCR and the 2(-Delta Delta C(T)) method. Methods 25: 402-408, 2001.

34. Sticht C, De La Torre C, Parveen A and Gretz N: miRWalk: An online resource for prediction of microRNA binding sites. PLoS One 13: e0206239, 2018.

35. Vilming Elgaaen B, Olstad OK, Haug KB, Brusletto B, Sandvik L, Staff AC, Gautvik KM and Davidson B: Global miRNA expression analysis of serous and clear cell ovarian carcinomas identifies differentially expressed miRNAs including miR-200c-3p as a prognostic marker. BMC Cancer 14: 80, 2014.
36. Paraskevopoulou MD, Georgakilas G, Kostoulas N, Reczko M, Maragkakis M, Dalamagas TM and Hatzigeorgiou AG: DIANA-LncBase: Experimentally verified and computationally predicted microRNA targets on long non-coding RNAs. Nucleic Acids Res 41(Database Issue): D239-D245, 2013.

37. Li JH, Liu S, Zhou H, Qu LH and Yang JH: starBase v2.0: Decoding miRNA-ceRNA, miRNA-ncRNA and protein-RNA interaction networks from large-scale CLIP-Seq data. Nucleic Acids Res 42 (Database Issue): D92-D97, 2014.

38. Chen G, Wang Z, Wang D, Qiu C, Liu M, Chen X, Zhang Q, Yan G and Cui Q: LncRNADisease: A database for long-non-coding RNA-associated diseases. Nucleic Acids Res 41 (Database Issue): D983-D986, 2013

39. Groeneweg JW, Foster R, Growdon WB, Verheijen RH and Rueda BR: Notch signaling in serous ovarian cancer. J Ovarian Res 7: 95, 2014

40. Khan A, Fornes O, Stigliani A, Gheorghe M, CastroMondragon JA, van der Lee R, Bessy A, Chèneby J, Kulkarni SR, Tan G, et al: JASPAR 2018: Update of the open-access database of transcription factor binding profiles and its web framework. Nucleic Acids Res 46: D1284, 2018.

41. Tang Z, Li C, Kang B, Gao G, Li C and Zhang Z: GEPIA: A web server for cancer and normal gene expression profiling and interactive analyses. Nucleic Acids Res 45: W98-W102, 2017.

42. Zhu H, Wang G, Zhou X, Song X, Gao H, Ma C, Chang H, Li H, Liu FF, Lu J and Ma J: miR-1299 suppresses cell proliferation of hepatocellular carcinoma (HCC) by targeting CDK6. Biomed Pharmacother 83: 792-797, 2016.

43. Liu LH, Tian QQ, Liu J, Zhou Y and Yong H: Upregulation of hsa_circ_0136666 contributes to breast cancer progression by sponging miR-1299 and targeting CDK6. J Cell Biochem 120: 12684-12693, 2019.

44. Meng L, Liu S, Ding P, Chang S and Sang M: Circular RNA ciRS-7 inhibits autophagy of ESCC cells by functioning as miR-1299 sponge to target EGFR signaling. J Cell Biochem 121: 1039-1049, 2020.

45. Morgan SL, Wyant GA and Dinulescu DM: 'Take it up a NOTCH': Novel strategies for cancer therapy. Cell Cycle 12: 191-192, 2013.

46. Sang M, Meng L, Liu S, Ding P, Chang S, Ju Y, Liu F, Gu L, Lian Y and Geng C: Circular RNA ciRS-7 maintains metastatic phenotypes as a ceRNA of miR-1299 to target MMPs. Mol Cancer Res 16: 1665-1675, 2018.

47. Shah MM, Zerlin M, Li BY, Herzog TJ, Kitajewski JK and Wright JD: The role of Notch and gamma-secretase inhibition in an ovarian cancer model. Anticancer Res 33: 801-808, 2013.

48. Chen X, Thiaville MM, Chen L, Stoeck A, Xuan J, Gao M, Shih IeM and Wang TL: Defining NOTCH3 target genes in ovarian cancer. Cancer Res 72: 2294-2303, 2012.

49. Young TL, Matsuda T and Cepko CL: The noncoding RNA taurine upregulated gene 1 is required for differentiation of the murine retina. Curr Biol 15: 501-512, 2005.

50. Guo S, Zhang L, Zhang Y, Wu Z, He D, Li X and Wang Z: Long non-coding RNA TUG1 enhances chemosensitivity in non-small cell lung cancer by impairing microRNA-221-dependent PTEN inhibition. Aging (Albany NY) 11: 7553-7569, 2019.

51. Yu G, Zhou H, Yao W, Meng L and Lang B: IncRNA TUG1 Promotes cisplatin resistance by regulating CCND2 via epigenetically silencing miR-194-5p in bladder cancer. Mol Ther Nucleic Acids 16: 257-271, 2019.

52. Zhang Z, Wang X, Cao S, Han X, Wang Z, Zhao X, Liu X, Li G, Pan and Lei D: The long noncoding RNA TUG1 promotes laryngeal cancer proliferation and migration. Cell Physiol Biochem 49: 2511-2520, 2018.

53. Barbagallo C, Brex D, Caponnetto A, Cirnigliaro $M$, Scalia M, Magnano A, Caltabiano R, Barbagallo D, Biondi A, Cappellani A, et al: LncRNA UCA1, upregulated in CRC biopsies and downregulated in serum exosomes, controls mRNA expression by RNA-RNA interactions. Mol Ther Nucleic Acids 12: 229-241, 2018.

54. He C, Liu Z, Jin L, Zhang F, Peng X, Xiao Y, Wang X, Lyu Q and Cai X: lncRNA TUG1-mediated miR-142-3p downregulation contributes to metastasis and the epithelial-to-mesenchymal transition of hepatocellular carcinoma by targeting ZEB1. Cell Physiol Biochem 48: 1928-1941, 2018.

55. Sun J, Hu J, Wang G, Yang Z, Zhao C, Zhang X and Wang J: LncRNA TUG1 promoted KIAA1199 expression via miR-600 to accelerate cell metastasis and epithelial-mesenchymal transition in colorectal cancer. J Exp Clin Cancer Res 37: 106, 2018. 
56. Xu Y, Ge Z, Zhang E, Zuo Q, Huang S, Yang N, Wu D, Zhang Y, Chen $\mathrm{Y}, \mathrm{Xu} \mathrm{H}$, et al: The lncRNA TUG1 modulates proliferation in trophoblast cells via epigenetic suppression of RND3. Cell Death Dis 8: e3104, 2017.

57. Kuang D, Zhang X, Hua S, Dong W and Li Z: Long non-coding RNA TUG1 regulates ovarian cancer proliferation and metastasis via affecting epithelial-mesenchymal transition. Exp Mol Pathol 101: 267-273, 2016.

58. Li TH, Zhang JJ, Liu SX and Chen Y: Long non-coding RNA taurine-upregulated gene 1 predicts unfavorable prognosis, promotes cells proliferation, and inhibits cells apoptosis in epithelial ovarian cancer. Medicine (Baltimore) 97: e0575, 2018.

59. Chen LL: Linking long noncoding RNA localization and function. Trends Biochem Sci 41: 761-772, 2016.

60. Ren K, Li Z, Li Y, Zhang W and Han X: Long noncoding RNA taurine-upregulated gene 1 promotes cell proliferation and invasion in gastric cancer via negatively modulating miRNA-145-5p. Oncol Res 25: 789-798, 2017.

61. Wang Y, Yang T, Zhang Z, Lu M, Zhao W, Zeng X and Zhang W: Long non-coding RNA TUG1 promotes migration and invasion by acting as a ceRNA of miR-335-5p in osteosarcoma cells. Cancer Sci 108: 859-867, 2017
62. Zhao L, Sun H, Kong H, Chen Z, Chen B and Zhou M: The lncRNA-TUG1/EZH2 axis promotes pancreatic cancer cell proliferation, migration and EMT phenotype formation through sponging miR-382. Cell Physiol Biochem 42: 2145-2158, 2017.

63. Sheng Q, Zhang Y, Wang Z, Ding J, Song Y and Zhao W: Cisplatin-mediated down-regulation of miR-145 contributes to up-regulation of PD-L1 via the c-Myc transcription factor in cisplatin-resistant ovarian carcinoma cells. Clin Exp Immunol 200: 45-52, 2020.

64. Tan H, He Q, Gong G, Wang Y, Li J, Wang J, Zhu D and Wu X: miR-382 inhibits migration and invasion by targeting ROR1 through regulating EMT in ovarian cancer. Int J Oncol 48: 181-190, 2016.

65. Zhu X, Li Y, Xie C, Yin X, Liu Y, Cao Y, Fang Y, Lin X, Xu Y, $\mathrm{Xu} \mathrm{W}$, et al: miR-145 sensitizes ovarian cancer cells to paclitaxel by targeting Sp1 and Cdk6. Int J Cancer 135: 1286-1296, 2014.

66. Katsushima K, Natsume A, Ohka F, Shinjo K, Hatanaka A, Ichimura N, Sato S, Takahashi S, Kimura H, Totoki Y, et al: Targeting the Notch-regulated non-coding RNA TUG1 for glioma treatment. Nat Commun 7: 13616, 2016.

This work is licensed under a Creative Commons Attribution-NonCommercial-NoDerivatives 4.0 International (CC BY-NC-ND 4.0) License. 